\title{
Akute mesenteriale Ischämie: Management und radiologische Interventionen in der Therapie akuter arterieller Verschlüsse und venöser Thrombosen
}

\author{
Jens-Christian Kröger Karlheinz Hauenstein \\ Institut für Diagnostische und Interventionelle Radiologie, Universität Rostock, Deutschland
}

\author{
Schlüsselwörter \\ Akute mesenteriale Ischämie · Rekanalisation . \\ Thrombolyse $\cdot$ Interventionelle Radiologie
}

\section{Zusammenfassung}

Die akute intestinale Ischämie (AMI) stellt trotz der in den letzten Jahren verbesserten diagnostischen Methoden auch heute ein Krankheitsbild mit hoher Mortalitätsrate dar. Die vorgestellte optimierte interdisziplinäre Therapie der AMI unter Einbeziehung aller interventionell-radiologischen Möglichkeiten ist nur bei Patienten mit frühzeitiger Diagnosestellung oder nach primärer chirurgischer Versorgung einsetzbar. In Abhängigkeit vom Typ der AMI kann sie die Bedingungen für eine Operation und letztendlich die Ergebnisse des chirurgischen Eingriffs verbessern. 22 Patienten mit unterschiedlichen Typen der AMI wurden mit verschiedenen radiologischen Interventionen behandelt, bei 13 Patienten erfolgte die Behandlung als Ersteingriff und bei 9 nach chirurgischer Resektion. Sechs Patienten hatten eine arterielle Embolie, 3 eine arterielle Thrombose, 5 eine nonokklusive mesenteriale Ischämie, 6 eine venöse Thrombose und 2 ein dissezierendes Aortenaneurysma. Trotz der positiven Selektion lag die Mortalität bei $27 \%$, da der Zeitpunkt irreversibler ischämischer Veränderungen der Darmwand nicht exakt zu bestimmen ist und eine verspätete Revaskularisation schwere oder letale Komplikationen verursacht. Die beste Prognose weisen Patienten auf, bei denen die AMI als Komplikation einer mesenterikoportalen Thrombose auftritt. Unser an den radiologischen Möglichkeiten orientiertes Konzept des diagnostischen und therapeutischen Managements optimiert die interdisziplinäre Zusammenarbeit und die Kombination chirurgischer und interventioneller Behandlungsformen.

\author{
Key Words \\ Acute intestinal ischemia - Recanalization . \\ Thrombolysis · Interventional radiology
}

\section{Summary}

Acute Mesenterial Ischemia: Management and Radiological Interventions in the Treatment of Acute Arterial Occlusion and Venous Thrombosis Although better diagnostic methods were developed in the last years acute mesenterial ischemia (AMI) actually is a disease with high mortality. The interdisciplinary treatment concept of AMI presented here includes various interventional radiologic methods and can be used in patients with early diagnosis of $A M I$ or after surgical bowel resection. Depending on the type of $\mathrm{AMI}$, the treatment should improve conditions and results of surgical treatment. 22 patients with different types of AMI were treated by various interventional radiologic procedures, 13 of them as primary intervention and 9 after bowel resection. In 6 patients AMI was caused by arterial embolism, in 3 by arterial thrombosis, in 5 by nonocclusive intestinal ischemia, in 6 by venous thrombosis and in 2 by aortic dissection. Although our patients are positively selected, we found a mortality of $27 \%$ because time of nonreversible ischemic damage of bowel wall cannot be defined exactly and late reperfusion leads to major or lethal complications. the best prognosis was shown for patients with mesenterico-portal thrombosis. Our concept of diagnostic and therapeutic management is orientated on interventional possibilities with the intention to improve interdisciplinary cooperation and optimize combination of surgical and interventional radiologic treatment.

\begin{tabular}{ll}
\hline KARGER & ( 2006 S. Karger GmbH, Freiburg \\
Fax +497614520714 & Accessible online at: \\
$\begin{array}{l}\text { E-mail Information@Karger.de } \\
\text { www.karger.com }\end{array}$ & www.karger.com/cga \\
&
\end{tabular}

Dr. med. Jens-Christian Kröger

Institut für Diagnostische und Interventionelle Radiologie Universität Rostock

Ernst-Heydemann-Straße 6, 18057 Rostock, Deutschland Tel. +49 381 49492-20, Fax -61

E-mail jens-christian.kroeger@med.uni-rostock.de 


\section{Einleitung}

Obwohl Patienten mit intestinalen Durchblutungsstörungen in den vergangenen Jahren gerade einmal 0,1\% der mit einem akuten chirurgischen Krankheitsbild aufgenommenen Patienten ausmachen [1, 2], steht der Komplex akuter mesenterialer Ischämien (AMI) immer wieder im Blickpunkt des Interesses der verschiedenen klinischen Fachrichtungen. Mit der weiter steigenden Lebenserwartung der Bevölkerung ist auch eine Zunahme des Auftretens der AMI zu prognostizieren, da mehr als $40 \%$ der Patienten mit akuten intestinalen Durchblutungsstörungen älter als 70 Jahre sind.

In den vergangenen Jahren musste trotz verbesserter diagnostischer Möglichkeiten und einer optimierten perioperativen Therapie konstatiert werden, dass kein signifikanter Rückgang der Mortalität bei Patienten mit AMI zu verzeichnen ist. Sie wird von unterschiedlichen Autoren über einen Zeitraum von mehr als 15 Jahren übereinstimmend mit 60-80\% angegeben [2-6]. Erwartungsgemäß lassen sich signifikante Unterschiede in der perioperativen Mortalität von über und unter 70-jährigen Patienten nachweisen [7].

Entscheidender prognostischer Faktor bleibt nach wie vor das Zeitintervall zwischen dem Auftreten der ersten klinischen Symptome und dem Therapiebeginn [8-10].

Der Schlüssel für eine Verringerung der Mortalität und der Minimierung der chirurgischen Resektion liegt daher im interdisziplinären Management von Diagnostik und Therapie. Im Interesse der schnellstmöglichen und wenig aufwendigen Diagnosestellung hat sich das Spektrum der diagnostischen Untersuchungen verändert. Die noch vor kurzem als Goldstandard betrachtete, zeitlich aufwendige und invasive angiographische Diagnostik wird heute in der Primärdiagnostik immer häufiger durch die Duplexsonographie und die Mehrzeilen-CT-Untersuchung mit intravenöser Kontrastmittelgabe ersetzt. Alternativ besteht auch die Möglichkeit der nichtinvasiven Diagnosesicherung mit Hilfe der MRT.

\section{Diagnostisches Management}

Die Effizienz des prätherapeutischen Managements wird in erster Linie vom zeitlichen Aufwand zwischen dem Auftreten der ersten Symptome und dem Beginn der Therapie bestimmt. Die Diagnosestellung wird durch das Fehlen eines charakteristischen Symptomkomplexes kompliziert. Wegweisend kann die Diskrepanz zwischen Schmerzen und abdominellem Untersuchungsbefund in Kombination mit einem Anstieg des Serum-Laktats über 2,4 mmol/1 sein. Der SerumLaktat-Wert besitzt im Gegensatz zu anderen Laborparametern wie CK-BB, LDH und alkalischer Phosphatase einen positiven Voraussagewert zur Erkennung der AMI. Die Erhöhung der D-Dimere ist zwar ebenfalls wenig spezifisch, kann aber auf das Vorliegen eines thrombotischen Prozesses hinweisen [11]. Veränderungen im Blutbild sind ebenfalls nur in Einzelfällen richtungsweisend, so z.B. bei myeloproliferativem Syndrom oder bei Verbrauchskoagulopathien. Die Leukozytose ist für eine Diagnosefindung zu unspezifisch, sie kann aber Grunderkrankungen oder das fortgeschrittene Ischämiestadium illustrieren. Gleiches gilt für die metabolische Azidose, die im spätem Stadium oder bei schwerer Begleitpathologie eintritt. Der zeitlich effektiven und ergebnisorientierten bildgebenden Diagnostik kommt daher eine besondere Bedeutung zu.

Aufgrund der breiten Verfügbarkeit und der schnellen Einsatzmöglichkeit kann man mit Hilfe der Sonographie, insbesondere durch die Einbeziehung der farbkodierten Duplexsonographie (FKDS), erste und frühe Zeichen der AMI diagnostizieren. So können Wandödem und Motilitätsänderungen betroffener Darmschlingen oder intraperitoneale Flüssigkeitsansammlungen nachgewiesen werden [12]. In der Duplexsonographie können Stenosen, arterielle Embolien oder Verschlüsse der Arterien ebenso erkannt werden wie Thrombosen der mesenterialen oder portalen Venen. Auch in der Beurteilung seltener Ursachen der mesenterialen Ischämie, wie dem dissezierenden Aortenaneurysma, kann die Sonographie bereits entscheidende Hinweise geben. Von Nachteil ist die stete Verschlechterung der Untersuchungsbedingungen mit Fortschreiten der AMI, die Beeinträchtigung der Untersuchung durch Darmgasansammlungen und die große Abhängigkeit von der Erfahrung des Untersuchers.

Die derzeit anerkannteste Methode der nichtinvasiven Diagnostik intestinaler Durchblutungsstörungen stellt die Mehrzeilen-Spiral-CT (MS-CT) mit intravenöser Kontrastmittelgabe dar $[13,14]$. Sie erlaubt mit dem geringsten zeitlichen Aufwand detaillierte Aussagen über die Beschaffenheit und die Perfusion der Darmschlingen, stenosierende und okklusive Veränderungen der intestinalen Arterien bis in die segmentalen Aufzweigungen, Thrombosen im mesenterikoportalen Venensystem, entzündliche oder Raum fordernde Begleiterkrankungen sowie Exsudationen und Gaseinschlüsse in der Darmwand und im portalvenösen System [15-17]. Sensitivität bzw. Spezifität der biphasischen MS-CT erreichen heute Werte von 96 bzw. 94\%. Die Technik bietet somit verlässliche direkte und indirekte Parameter zur Beurteilung des intestinalen Perfusionsstatus und der Ursachen einer AMI [13, 18].

Die MRT kann derzeit trotz des hohen Entwicklungsstands der MR-Angiographie nicht als Standardmethode zur Untersuchung der AMI angesehen werden, da sie einen kooperativen Patienten voraussetzt und gleichzeitig Einschränkungen im Patienten-Monitoring erfordert [19, 20]. Außerdem ist die Differenzierung zwischen frühen ischämischen, infiltrativen und entzündlichen Veränderungen im MRT schwierig, erfordert unterschiedliche Sequenzen und erscheint damit in Bezug auf den Zeitaufwand vergleichsweise ungünstig.

Der Einsatz der digitalen Subtraktionsangiographie (DSA) erfolgt meist erst zur weiterführenden Diagnostik oder im Rahmen eines interventionellen Eingriffs. Lediglich in der Beurteilung umschriebener peripherer Embolien und der nicht- 

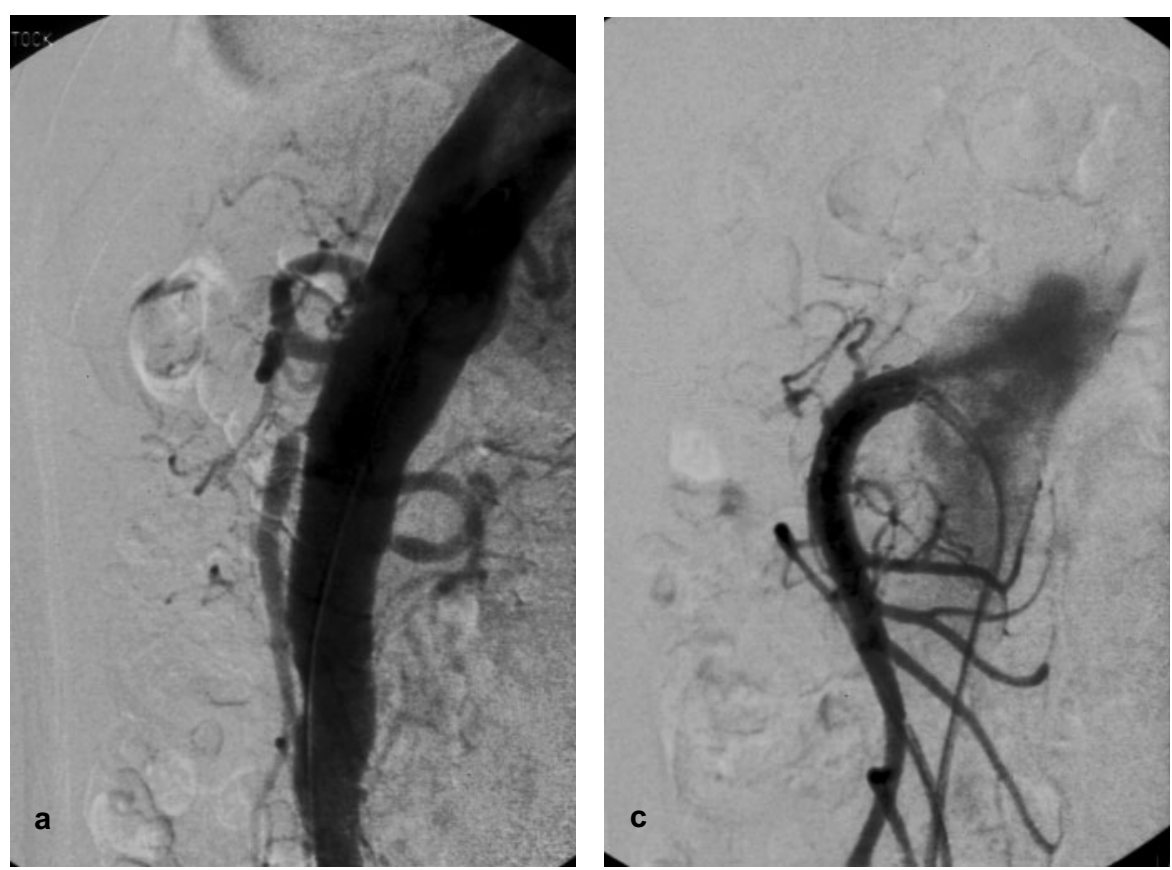

Abb. 1. a 72-jähriger Patient mit AMI durch periphere Embolien bei hochgradiger Abgangsstenose der A. mesenterica superior und

Verschluss der Arteria mesenterica inferior.

Kontrolle der Platzierung eines ballonexpandiblen Stents über die Schleuse b vor und $\mathbf{c}$ nach Balloninflation. d Übersichtsangiographie nach Beseitigung der Stenose.
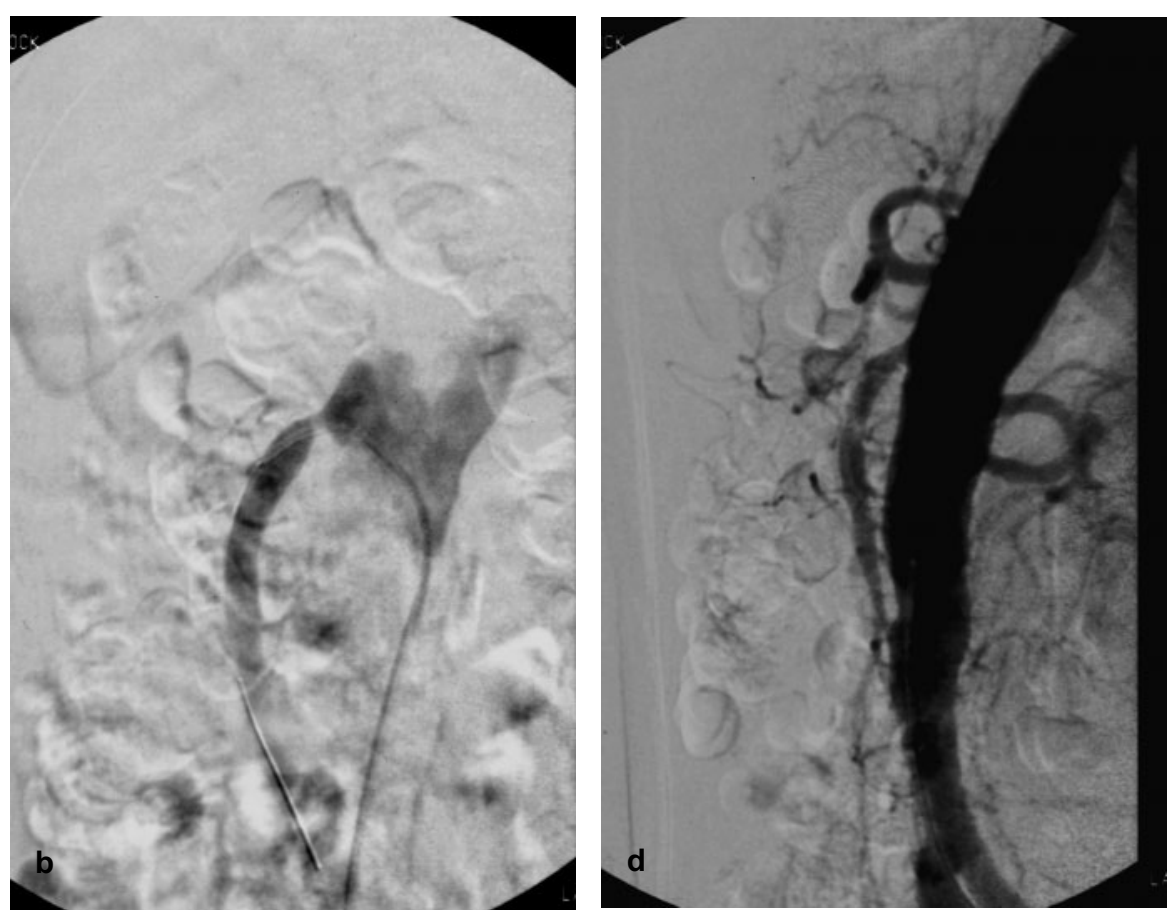

okklusiven mesenterialen Ischämie (NOMI) ist die selektive Angiographie der Mesenterialarterien allen anderen diagnostischen Verfahren überlegen.

Aufgabe der Diagnostik ist nicht nur der Nachweis, sondern vor allem die Differenzierung der verschiedenen Typen der AMI. Von ebenso großer Bedeutung ist der Nachweis irreversibler ischämischer Veränderungen der Darmwand, da diese einen primären chirurgischen Eingriff erfordern. Außerdem sollten mögliche therapierelevante kausale Zusammenhänge wie z.B. eine akute Aortendissektion, Ileussituationen sowie entzündliche oder tumoröse Veränderungen erkannt werden, um eine effiziente Soforttherapie einleiten zu können.

\section{Therapiealgorithmus bei AMI}

Es werden 4 Grundtypen der AMI unterschieden:

- arterielle Embolie,

- arterielle Thrombose,

- NOMI,

- venöse Thrombose.

Zusätzlich sind zwei Sonderformen der AMI zu erwähnen: - nonokklusive AMI bei dissezierendem Aortenaneurysma, - vaskulitisassoziierte Mesenterialvenenthrombose [21].

Die Angaben verschiedener Autoren bezüglich der Häufigkeit der einzelnen Typen variieren erheblich. Übereinstimmend 
kann lediglich festgestellt werden, dass die arterielle Embolie etwa $60-70 \%$ und die arterielle Thrombose etwa $20-25 \%$ der Fälle von AMI verursacht. Der prozentuale Anteil der NOMI schwankt in den Angaben zwischen 2 und 10\%, hier beeinflusst vor allem das chirurgische und intensivmedizinische Profil einer Einrichtung die Häufigkeit. In zirka 10\% der Fälle wird die AMI durch eine Thrombose der mesenterialen und portalen Venen hervorgerufen.

Der Therapiealgorithmus in unserer Einrichtung orientiert sich an zwei wegweisenden Faktoren: am Typ der AMI und am Nachweis irreversibler Perfusionsstörungen der Darmwand. Während die arterielle Embolie und die Thrombose im Regelfall operativ versorgt werden, können die NOMI und die Thrombose der mesenterikoportalen Venen bei fehlenden Zeichen einer Gangrän einer primären interventionellen Therapie unterzogen werden. Bei einer AMI als Komplikation einer Aortendissektion hängt das Vorgehen davon ab, ob eine thrombotische Obliteration der Arteria mesenterica superior vorliegt.

\section{Arterielle Embolie oder Thrombose}

Bei Nachweis einer AMI auf dem Boden einer arteriellen Embolie oder Thrombose streben wir die primäre chirurgische Rekanalisation an, bei Vorliegen einer Gangrän erfolgt die Resektion der betroffenen Darmsegmente.

Bestehen intraoperativ Perfusionsdefizite, die auf inkomplette oder periphere arterielle Restokklusionen schließen lassen, eine Resektion jedoch nicht rechtfertigen, folgt unmittelbar auf den chirurgischen Eingriff eine DSA mit Option der Intervention [22]. Nach Möglichkeit wird der transfemorale $\mathrm{Zu}$ gang gewählt, da bei transbrachialem Vorgehen interventionelle Eingriffe erschwert sein können, Schleusensysteme in ihrem Durchmesser limitiert sind und eine Langzeit-Katheterplatzierung für Dauerinfusionen das Risiko einer Embolie in den supraaortalen Arterien mit sich bringt. Für die Beurteilung der viszeralen Kollateralsysteme sollte stets eine Darstellung beider Mesenterialarterien und des Truncus coeliacus angestrebt werden. Ob für die Optimierung der Darstellung kleiner Gefäße Scopolamin (intraarteriell) oder Glukagon (intravenös) eingesetzt werden können, hängt von der Kreislaufsituation und Begleiterkrankungen (Diabetes mellitus; Glaukom) ab. Kritische Stenosen bzw. kurzstreckige Verschlüsse der Mesenterialarterien oder auch des Truncus coeliacus werden durch eine primäre Stent-Implantation beseitigt. Das technische Vorgehen bei stenoseassoziierter AMI unterscheidet sich nicht von dem bei chronischer intestinaler Ischämie. Über eine $50 \mathrm{~cm}$ lange renal konfigurierte $6 \mathrm{~F}-\mathrm{Schleuse}$ mit heparinisierter Dauerspülung wird ein 4F-Katheter vom Typ Cobra oder Sidewinder direkt vor der Stenose oder dem Verschluss platziert. Verschlüsse werden primär mit einem hydrophil beschichteten Führungsdraht vorsichtig passiert, anschließend wird mit Schraubbewegungen der Katheter darüber geschoben. Bei besonders elongiertem Gefäßverlauf kann diese Prozedur gegebenenfalls auch in mehreren Stufen und unter Zuhilfenahme eines 2,3F-Mikrokathetersystems vorgenommen werden. Die Passage des Verschlusses ermöglicht dann das Einlegen eines entsprechend der Gefäßanatomie vorgebogenen steifen Interventionsführungsdrahts, der eine ausreichende Stabilität für das nachfolgende Einbringen eines Ballonkatheters zur Vordilatation der Verschlussstrecke bietet. Bei kurzstreckigen Stenosen oder Okklusionen erreicht man durch die Implantation ballonexpandibler Stents mit einer Länge von 7-24 mm gute Ergebnisse (Abb. 1). Von Vorteil sind dabei die präzise Platzierbarkeit, die durch Injektionen von Kontrastmittel über die vor dem Ostium der Arterie liegende Schleuse kontrolliert werden kann, und die problemlose Beseitigung stark kalzifizierter Stenosen. Wir bevorzugen wegen des einfacheren Handlings «Rapid-exchange»-Systeme. Bei hochgradigen Stenosen, sehr rigiden Verschlüssen oder ausgeprägten Auslenkungen der Gefäßachse kann das Vorgehen in «Over-the-wire»-Technik allerdings bessere Vorschubbedingungen bieten. Bei den selten vorkommenden längeren Stenosen der A. mesenterica superior können sich selbstexpandierende Stents aufgrund ihrer größeren Flexibilität besser an die Gefäßmotilität anpassen [23]. Sie müssen dann so kurz wie möglich und mit einem «oversizing» des Arteriendurchmessers von 1-2 mm gewählt werden.

Bei den seltener vorkommenden Thrombosen der A. mesenterica superior berichten einzelne Autoren über erfolgreiche lokale Applikation von rekombinantem Gewebeplasminogenaktivator (rtPA) und Urokinase [24].

Sind im mesenterialen Stromgebiet spastische Engstellungen peripherer Arterien erkennbar, besteht die Indikation zur vasodilatativen Therapie [25]. Die lokale Infusion von Papaverin oder Prostaglandin E1 über einen selektiv in der A. mesenterica superior platzierten $4 \mathrm{~F}-$ Katheter bei gleichzeitiger systemischer Heparinisierung kann zu einer Minimierung der erforderlichen Resektionslänge beitragen. Das Vorgehen erfolgt analog zur nachstehend beschriebenen Behandlung der NOMI. Nach einer maximalen Infusionsdauer von $48 \mathrm{~h}$, die unter intensivmedizinischen Bedingungen und ständiger Beobachtung der Laborwerte, aber ohne angiographische Verlaufskontrollen erfolgt, wird ein chirurgischer «Second look»Eingriff vorgenommen.

\section{NOMI}

Im Gegensatz zu den anderen Formen der AMI tritt die NOMI nicht selten in der postoperativen Phase nach größeren chirurgischen Eingriffen auf. In der Häufigkeit dominieren kardiochirurgische Eingriffe, die NOMI wird aber auch nach gefäß- oder viszeralchirurgischen Eingriffen beobachtet [18, 19]. Hier lösen «Low output»-Syndrom, Hypovolämie, hypotone Kreislaufdysregulation, Katecholamin-Gaben und die damit verbundene Sympathicus-Aktivierung eine Autoregulationsstörung der intestinalen Gefäße aus, die zu einer periphe- 
Tab. 1. Medikamentenapplikation zur lokalen Therapie der NOMI

\begin{tabular}{lllll}
\hline Medikament & $\begin{array}{l}\text { Bolus, } \\
\mu \mathrm{g}\end{array}$ & $\begin{array}{l}\text { Infusionsdosis, } \\
\mu \mathrm{g} / \mathrm{h}\end{array}$ & $\begin{array}{l}\text { Infusionsdauer, } \\
\mathrm{h}\end{array}$ & Kontraindikationen \\
\hline Prostaglandin E1 & 20 & 2,5 & maximal 48 & $\begin{array}{l}\text { Gangrän } \\
\text { Peritonitis } \\
\text { akutes Blutungsrisiko } \\
\text { akuter Myokardinfarkt } \\
\text { akuter Apoplex } \\
\text { Lungenödem } \\
\text { Leberinsuffizienz }\end{array}$ \\
& & & \\
& & & Gangrän \\
& & & $\begin{array}{l}\text { Peritonitis } \\
\text { Apoplex }\end{array}$ \\
Papaverin & & & Leberinsuffizienz \\
& \multirow{2}{*}{10000} & $40000-60000$ & maximal 72 & \\
& & & \\
& & &
\end{tabular}

ren Widerstandserhöhung, einer Gegenregulation mit Tonuszunahme und damit zu einem persistierenden Spasmus führen. Verstärkend wirken Digitalis-Präparate, Gewebetoxine und vasokonstriktive Substanzen, die im Rahmen der perioperativen Intensivtherapie Einsatz finden. Auch die Reperfusion des ischämischen und infizierten Gewebes kann eine NOMI auslösen [20]. Ein Auftreten der NOMI ist bei Patienten mit Niereninsuffizienz und Hämodialyse, bei Behandlung mit Ergotamin-Derivaten oder bei schwerer kardialer Insuffizienz mit stark reduzierter Ejektionsfraktion beobachtet worden. [19].

Bereits der Verdacht auf eine NOMI rechtfertigt das Umgehen anderer, weniger aussagekräftiger diagnostischer Schritte und die unmittelbare angiographische Untersuchung, da sie als einziges Verfahren die Diagnose sichern kann. Der wesentliche Vorteil liegt in der Möglichkeit des unverzüglichen Übergangs zur Therapie. Voraussetzung ist allerdings auch hier, dass zum Zeitpunkt des Therapiebeginns keine Peritonitis besteht, da das Reperfusionstrauma bei bestehender Gangrän fast immer im Schock oder im Multiorganversagen endet [26, 27].

Nach Angiographie mit einem transfemoral eingebrachten 4F-Selektiv-Katheter und Nachweis der NOMI muss eine stabile Positionierung des Katheters erreicht werden. Gleichzeitig soll der Katheter bei einer möglichen Liegezeit von bis zu 72 h keine Gefäßwandirritationen verursachen. Es kann daher erforderlich sein, den Diagnostikkatheter der DSA gegen einen anderen Katheter auszutauschen. Alternativ ist auch die Platzierung eines 2,1F-Mikrokatheters möglich, wobei der Diagnostikkatheter als Führungskatheter dient. Wichtig ist die Prophylaxe thrombotischer Komplikationen, die durch die systemische Heparin-Gabe und, falls erforderlich, durch permanente Druckspülungen der Schleuse und des Führungs katheters gewährleistet wird.

In der klinischen Praxis haben sich als vasodilatative Pharmaka zur Behandlung der NOMI nur Papaverin und Prostavasin durchsetzen können. Der Einsatz anderer Medikamente wie
z.B. Captopril beschränkt sich auf tierexperimentelle Studien [28], weitere Pharmaka zur Behandlung der NOMI wie z.B. Tolazolin sind nicht mehr verfügbar.

Papaverin als Parasympathikolytikum führt zu einer Relaxation der glatten Muskulatur in der Gefäßwand. Der systemische Effekt der Blutdrucksenkung ist zu vernachlässigen, problematisch können aber hochfrequente Tachykardien oder Arrhythmien sein. Bei Intensivüberwachung ist eine Korrektur dieser Nebenwirkungen durch Verringerung der Infusionsdosis zu steuern.

Prostaglandin E1 hat neben seiner vasodilatativen Wirkung auch noch den Vorteil einer lokalen Einwirkung auf das Gerinnungssystem. Es ist in der Lage, den Plasminogenaktivator und damit die Fibrinolyse zu stimulieren, außerdem hemmt es die Thrombozyten-Aggregationsfähigkeit und verbessert die Restitution der Mikrozirkulation. Als Nebenwirkungen sind neben der Blutdrucksenkung und Tachykardie vor allem Kopfschmerzen und Durchfall in individuell stark variierender Intensität zu erwähnen.

Die für die Therapie empfohlene Dosierung der Pharmaka und die zu beachtenden Kontraindikationen sind in Tabelle 1 zusammengefasst.

Die Infusion der Vasodilatantien erfolgt unter intensivmedizinischen Bedingungen. Die Begleittherapie umfasst neben der partiellen-Thromboplastinzeit(PTT)-wirksamen Heparinisierung die Volumensubstitution, die Gewährleistung konstanter Sauerstoff-Sättigung und, im Bedarfsfall, den Ausgleich einer Azidose. Inwieweit man durch die Antioxydantien-Therapie eine Verbesserung der Prognose erreicht, bedarf weiterer Abklärung [29, 30].

Bei Anzeichen von Peritonitis ist eine Resektion der gangränös veränderten Darmschlingen unumgänglich. Die lokale Kathetertherapie der NOMI sollte keinesfalls abgesetzt werden, da der chirurgische Eingriff die spastischen Gefäßreaktionen zugleich wieder intensivieren kann. Eine Verlängerung der empfohlenen Infusionsdauer ist nur im Einzelfall und mit niedrigeren Dosierungen sinnvoll, wenn es sich um 
eine eine Katecholamin-induzierte NOMI bei intensivmedizinisch kritischen Patienten mit einer rechtfertigenden Prognose handelt.

\section{Thrombose der mesenterialen und portalen Venen}

Thrombosen der mesenterialen und portalen Venen lösen nur bei fulminantem Verlauf eine Ischämie der Darmwand aus. In einem Zeitraum von 10 Jahren wurden an unserer Einrichtung nur 5 von insgesamt 46 Pfortaderrekanalisationen mit der Indikation einer akuten intestinalen Durchblutungsstörung durchgeführt.

Die ursächlichen Faktoren sind vielfältig: entzündliche Darmerkrankungen, chirurgische Eingriffe wie Lebertransplantation oder Splenektomie, Leberparenchymerkrankungen oder das Budd-Chiari-Symdrom können eine mesenteriale Thrombose auslösen [31-34]. Auch das myeloproliferative Syndrom, die Sichelzellanämie oder Defizite der Faktoren C und S wurden als Ursache einer AMI auf der Basis einer mesenterialen Thrombose beobachtet [35-37].

Die therapeutische Strategie wird auch bei der venösen Thrombose durch das Fehlen oder den Nachweis einer irreversiblen Darmschädigung bestimmt. Bei bestehenden Zeichen einer Gangrän oder Peritonitis wird primär die Resektion der betroffenen Darmschlingen vorgenommen. Intraoperativ wird ein 4F-Katheter mit einem Lumen von 0,038-0,042 Zoll in ein periphere Vene eingebracht und fixiert. Dieser Katheter dient als Zugang für eine lokoregionale, niedrig dosierte Lysetherapie, kann aber im Weiteren als «Introducer» für ein 2,3FMikrokathetersystem genutzt werden. Dadurch bietet sich die Möglichkeit, die Thrombolyse nach retrograder Katheterisierung weiterer mesenterialer Venen auch auf andere Abschnitte des mesenterialen Venensystems auszudehnen.

Die zweite Methode, die wir sowohl als radiologische Primärintervention bei fehlenden Zeichen einer Peritonitis als auch im Rahmen des Sekundäreingriffs nach erfolgter Darmresektion einsetzen, basiert auf der transjugulär-transhepatischen Punktion eines intrahepatischen Pfortaderasts mit anschlieBender retrograder Rekanalisation thrombosierter portaler und mesenterialer Venen. Die Punktion wird analog zur Anlage eines transjugulären intrahepatischen portosystemischen Stent-Shunts (TIPS) unter sonographischer Steuerung mit der modifizierten Ross-Nadel durchgeführt (Abb. 2). Bei frischen Thrombosen der portalen Äste ist deren sonographische Abgrenzbarkeit nicht eingeschränkt, da sich die Thromben echoarm darstellen. Favorisiert wird die Punktion des rechten Pfortaderhauptasts, da nachfolgende Interventionen in einer günstigen Zugangsachse durchgeführt werden können. Die Passage der thrombosierten Venen wird auf möglichst atraumatische Weise mit einem Führungsdraht mit zurückgezogener Seele oder einem hydrophil beschichteten Führungsdraht mit $8 \mathrm{~cm}$ langem flexiblem Segment durchgeführt. Hierüber wird eine $80 \mathrm{~cm}$ lange $8 \mathrm{~F}$-Schleuse mit «Multipurpose»-Konfi-
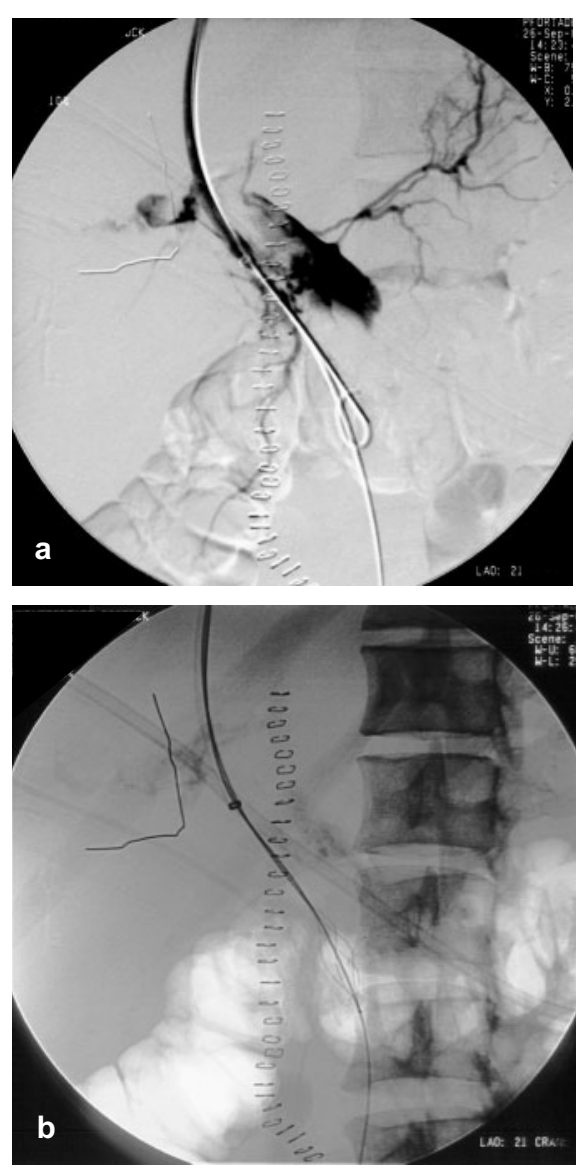

Abb. 2. 44-jährige Patientin mit AMI und primärer Resektion einer Jejunalschlinge bei kompletter mesenterikoportaler Thrombose nach Splenektomie. Transjugulär-transhepatische Thrombusfragmentation mit a Ringkatheter und $\mathbf{b}$ Basket. Dreitägige lokale Thrombolyse, Aspirationsthrombektomie und Rekanalisation der Vena mesenterica superior, der Pfortader und rechtshepatischer portaler Äste (c, d).Keine Anlage eines temporären TIPS.

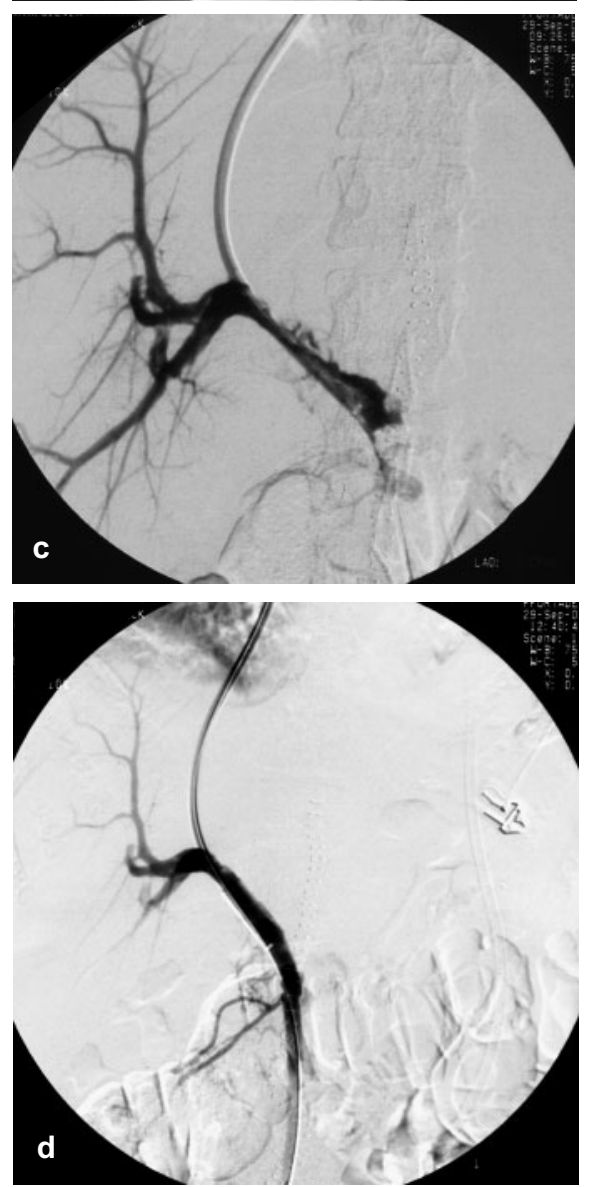


Tab. 2. Eigene Ergebnisse der radiologischinterventionellen Therapie bei AMI

\begin{tabular}{llllll}
\hline & $\begin{array}{l}\text { Arterielle } \\
\text { Embolie }\end{array}$ & $\begin{array}{l}\text { Arterielle } \\
\text { Thrombose }\end{array}$ & NOMI & $\begin{array}{l}\text { Venöse } \\
\text { Thrombose }\end{array}$ & $\begin{array}{l}\text { Aorten- } \\
\text { dissektion }\end{array}$ \\
\hline Patientenzahl & 6 & 3 & 5 & 6 & 2 \\
Primärintervention & 2 & 1 & 3 & 5 & 2 \\
Technischer Erfolg & 2 & 1 & 2 & 5 & 2 \\
Komplikationen & - & 1 Blutung & 2 Organversagen & - & - \\
Nachresektionen & 1 & - & 2 & - & 1 \\
Mortalität & 1 & 1 & 2 & - & - \\
Sekundärintervention & 4 & 2 & 2 & 1 & - \\
Technischer Erfolg & 4 & 2 & 1 & 1 & - \\
Komplikationen & - & - & 1 Organversagen & - & - \\
Mortalität & - & 1 & 1 & - & - \\
\hline
\end{tabular}

guration und abnehmbarem hämostatischem Ventil bis in den Pfortaderstamm vorgebracht. Über diese Schleuse können alle nachfolgenden interventionellen Prozeduren gezielt realisiert werden, die Spitzenkonfiguration gestattet eine Steuerung.

Zur mechanischen Alteration der Thromben in der Pfortader, im mesenterialen Venenstamm und in der Vena lienalis werden rotierende Ringkatheter, großlumige Ballonkatheter oder «Baskets» eingesetzt. Die Thrombektomie kann mit Hilfe von Aspirationskathetern oder Thrombektomiesystemen wie dem Amplatz-Device erfolgen. Von entscheidender Bedeutung für die anschließende Thrombolyse ist die Restitution der hepatopetalen Flussrichtung in der Pfortader. Über einen in der mesenterialen Peripherie platzierten 4F-Katheter wird das Thrombolytikum appliziert, über die Schleuse läuft gleichzeitig die lokoregionale Heparin-Applikation. Wir verwenden für die Thrombolyse rtPA (Actilyse ${ }^{\circledR}$, Boehringer Ingelheim Pharma GmbH \& Co. KG, Ingelheim am Rhein, Deutschland) mit einem Bolus von $5 \mathrm{mg}$ und einer Infusionsdosis von $2,5 \mathrm{mg} / \mathrm{h}$ bei einem Dosismaximum von $40 \mathrm{mg} / 24 \mathrm{~h}$ oder Urokinase mit einem Bolus von $50000 \mathrm{U}$ und einer Infusionsdosis von $40000 \mathrm{U} / \mathrm{h}$ bei einer Gesamtdosis von $800000 \mathrm{U} / 24 \mathrm{~h}$. Die begleitende Heparin-Therapie wird auf eine Ziel-PTT von 50-60 s ausgerichtet. Die Thrombolyse erfolgt mit laborchemischem und intensivmedizinischem Monitoring. Radiologische Kontrollen mit zusätzlichen rekanalisierenden Interventionen und Katheterneuplatzierung erfolgen nach 12 und $24 \mathrm{~h}$. Erst nach $24 \mathrm{~h}$ wird in Abhängigkeit von der portalen Hämodynamik die Anlage eines temporären TIPS in Erwägung gezogen. In den meisten Fällen mit frischer Thrombose gelingt die simultane extra- und intrahepatische Thrombolyse der portalen Strombahn. Bei mehrzeitig entstandenen Thrombosen mit älteren intrahepatischen Anteilen kann es erforderlich sein, zuerst einen TIPS mit geringem Durchmesser zu installieren, um die Vorteile einer portalen Drucksenkung für die Regeneration der Darmwand nutzen zu können. Erst nach Behebung der kritischen portalen Abflussbeeinträchtigung und Wiederherstellung der Durchgängigkeit der extrahepatischen Zuflüsse widmet man sich dann der Rekanalisation der intrahepatischen Pfortaderäste. Lediglich bei der durch ein
Budd-Chiari-Syndrom verursachten AMI ist die Anlage eines TIPS vor Beginn der peripheren Thrombolyse zwingend erforderlich.

\section{Sonderformen der AMI}

Bei akut eintretender mesenterialer Ischämie als Komplikation eines dissezierenden Aortenaneurysmas besteht die Möglichkeit einer Fensterung der Dissektionsmembran. Nach Möglichkeit sollte die Fensterung auf Höhe der Abgänge des Truncus coeliacus oder der A. mesenterica superior erfolgen, um einen suffizienten Druckanstieg im Darm versorgenden Lumen zu erreichen. Auch hier besteht die Einschränkung, dass bei Zeichen der irreversiblen Darmschädigung der chirurgische Eingriff Vorrang hat.

Für die Fensterung wird über eine transfemoral platzierte, 23 cm lange 8F-Schleuse eine Transseptalnadel in einem schützenden Führungskatheter eingebracht. Gleichzeitig katheterisiert man das zweite Lumen über einen transbrachialen oder kontralateralen femoralen Zugang mit einem 6F-Katheter. Unter Durchleuchtungskontrolle oder Kontrolle mittels intravasaler Sonographie wird die Membran gezielt durchstochen, ein interventioneller Führungsdraht wird in das zweite Lumen vorgeschoben. Über diesen Draht kann dann ein Ballonkatheter von $4 \mathrm{~cm}$ Länge und $5 \mathrm{~mm}$ Durchmesser zur Dilatation der Perforationsstelle genutzt werden. Danach wird ein großlumiger Ballon mit 18-26 mm Durchmesser zur Dilatation eines ausreichend großen Wiedereintritts genutzt. Diese Nachdilatation kann aber auch durch eine Stent-Fixation der Membran am Ostium der Arterie ersetzt werden, wenn nur eine der unpaaren Oberbaucharterien durchgängig ist. Werden mehrere Arterien aus diesem Lumen versorgt, ist der Fensterung der Vorrang zu geben.

\section{Eigene Behandlungsergebnisse}

Die Ergebnisse unserer radiologisch-interventionell oder chirurgisch- und radiologisch-interventionell behandelten Patien- 
ten mit AMI spiegeln das eigentliche Dilemma der Therapie der AMI nicht wider, da es sich um ein positiv selektioniertes Patientengut handelt. Trotzdem kann man in der interdisziplinären Versorgung den Ansatz zur Optimierung der bisher oft fatalen Behandlungsergebnisse sehen (Tab. 2). Die Mortalität liegt mit 27\% weit unter den Angaben für die gesamte Patientengruppe mit zirka $60 \%$. Intestinale Ischämien auf der Basis von venösen Thrombosen und Aortendissektionen haben bei rechtzeitiger Diagnose eine günstigere Prognose, auch nach Resektion steht ein effektives interdisziplinäres Behandlungskonzept zur Verfügung. Die große Bedeutung einer rechtzeiti- gen Therapie wird dadurch unterstrichen, dass bei 4 von 22 Patienten ein letaler Ausgang mit der massiven Einschwemmung von Toxinen aus ischämisch vorgeschädigten und interventionell revaskularisierten Darmabschnitten zu beobachten war. Dieses Risiko wird durch die präinterventionelle Resektion zwar verringert, aber nicht beseitigt.

Die technischen Komplikationsraten der interventionellen Eingriffe sind bei entsprechender Expertise der interventionellen Radiologen niedrig und unterscheiden sich nicht von denen bei analogen Interventionen in anderen Patientenkollektiven.

\section{Literatur}

1 Endean ED, Barnest SL, Kwolek CJ, Minion DJ, Schwarcz TH, Mentzer RM: Surgical management of thrombotic acute intestinal ischemia. Ann Surg 2001;233:801-98.

2 Raudonaitis A, Kavaliauskas K, Krimelis A: Extraordinary revascularization of acute-on-chronic intestinal ischemia. Medicina (Kaunas) 2002;38: $730-7$.

3 McKinsey JF, Gewertz BL: Acute mesenterial ischemia. Surg Clin North Am 1977;77:307-18.

4 Tendler DA: Acute intestinal ischemia and infarction: Semin Gastrointest Dis 2003;14:66-76.

$\checkmark 5$ Paes E, Vollmar JF, Hutschenreiter S, Schoenberg MH, Kubel R, Scholzel E: Der Mesenterialinfarkt. Neue Aspekte in Diagnostik und Therapie. Chirurg 1988;59:828-35.

6 Lock G: Acute mesenteric ischemia: classification, evaluation and therapy. Acta Gastroenterol Belg 2002;65:220-5.

7 Ritz JP, Runkel N, Berger G, Buhr HJ: Prognostische Faktoren beim Mesenterialinfarkt. Zentralbl Chir 1997;122:332-8.

8 Lock G: Acute intestinal ischemia. Best Pract Res Clin Gastroenterol 2001;15:83-8.

9 Park WM, Glowiczki P, Cherry KJ Jr, Hallett JW Jr, Bower TC, Panneton JM, Schleck C, Ilstrup D, Harmsen WS, Noel AA: Contemporary management of acute mesenteric ischemia: factors associated with survival. J Vasc Surg 2002;35:445-52.

10 Luther B, Moussazadeh K, Müller BT, Franke C, Harms J-M, Ernst S, Sandmann W: Die akute mesenteriale Ischämie - unverstanden oder unheilbar? Zentralbl Chir 2002;127:674-84.

11 Acosta S, Nilsson TK, Bjorck M: Preliminary study of $\mathrm{D}$-dimer as a possible marker of acute bowel ischemia. Br J Surg 2001;88:385-8.

12 Hidajat N, Stobbe H, Griesshaber V, Schröder RJ, Felix R: Portal vein thrombosis: etiology, diagnostic strategy, therapy and management. Vasa 2005;34: 81-92.

13 Wiesner W, Steinbrich W: Stellenwert der Computertomographie bei der Diagnostik der akuten Darmischämie. Radiologie up2date 2004;4:75-85.

14 Horton KM, Fishman EK: Multi-detector row CT of mesenteric ischemia: can it be done? Radiographics 2001;21:1463-73.
15 Haage P, Krings T, Schmitz-Rode T: Nontraumatic vascular emergencies: imaging and intervention in acute venous occlusion. Eur Radiol 2002;12: 2627-43.

16 Mallo RD, Salem L, Lalani T, Flum DR: Computed tomography diagnosis of ischemia and complete obstruction in small bowel obstruction: a systematic review. J Gastrointest Surg 2005;9:690-4.

17 Bradbury MS, Kavanagh PV, Chen MY, Weber TM, Bechtold RE: Noninvasive assessment of portomesenteric venous thrombosis: current concepts and imaging strategies. J Comput Assist Tomogr 2002;26:392-404.

18 Kirkpatrick ID, Kroeker MA, Greenberg HM: Biphasic CT with mesenteric CT angiography in the evaluation of acute mesenteric ischemia: initial experience. Radiology 2003;229:91-8.

19 Krämer SC, Görich J, Oertel F, Scheld H, Heindel W: Non-okklusive Darmischämie: Radiologische Diagnostik und Therapie. Fortschr Röntgenstr 2003;175:1177-83.

20 Ernst S, Luther B, Zimmermann N, Böhner H, Wilke R, Feindt, P, Fürst G: Aktuelle Diagnostik und Therapie der nicht okklusiven mesenterialen Ischämie. Fortschr Röntgenstr 2003;175:515-23.

21 Clark RA, Gallant TE: Acute mesenteric ischemia: angiographic spectrum. Am J Roentgenol 1984;142: 555-62.

22 Resch T, Lindh M, Dias N, Sonesson B, Uher P, Malina M, Ivancev K: Endovascular recanalisation in occlusive mesenteric ischemia - feasibility and early results. Eur J Vasc Endovasc Surg 2005;29: 199-203.

23 Waggershauser T: Mesenterialarterien; in Görich J, Brambs HJ (Hrsg): Interventionelle minimal invasive Radiologie. Stuttgart, Thieme 2001, pp173175 .

24 Hommann M, Richter KK, Heyne J, Behrend U, Rimpler H, Will U, Bartel M, Scheele J: Lysetherapie bei arterieller Thrombose der Arteria mesenterica superior. Zentralbl Chri 2003;128:155-8.

25 Kozuch PL, Brandt LJ: Review article: diagnosis and management of mesenteric ischaemia with an emphasis on pharmacotherapy. Aliment Pharmacol Ther 2005;21:201-15.

26 Pierro A, Eaton S: Intestinal ischemia reperfusion injury and multisystem organ failure. Semin Pediatr Surg 2004;13:11-17.
27 Stöckmann H, Roblick UJ, Kluge N, Kunze U, Schimmelpenning H, Kujath P, Müller G, Bruch HP: Diagnostik und Therapie der nicht okklusiven Mesenterialischämie (NOMI). Zentralbl Chir 2000; 125:144-51.

28 Jakschik, J, Jaeger U: Interventionelle Therapie der nonokklusiven mesenterialen Ischämie mit Captopril - eine tierexperimentelle Untersuchung. Fortschr Röntgenstr 1996;164:502-6.

29 Cuzzocrea S, Riley DP, Caputi AP, Salvemini D: Antioxydant therapy: a new pharmacological approach in shock, inflammation and ischemia/reperfusion injury. Pharmacol Rev 2001;53:135-59.

30 Alvarez B, Radi R: Peroxynitrite decay in the presence of hydrogene peroxide, mannitol and ethanol: a reappraisal. Free Radic Res 2001;34:467-75.

31 Kröger J, Schulze CG, Schareck WD, Klar E, Hauenstein K: Management of mesenteric ischemia due to arterial or venous occlusion. Cardiovasc Intervent Radiol 2004;27(suppl 1):133.

32 Hidajat N, Stobbe H, Griesshaber V, Schröder RJ, Felix R: Portal vein thrombosis: etiology, diagnostic strategy, therapy and management. Vasa 2005;34: 81-92.

33 Blum U, Rössle M, Haag, K, Ochs A, Blum HE, Hauenstein KH, Astinet F, Langer M: Budd-Chiari syndrome: technical, hemodynamic and clinical results of treatment with transjugular intrahepatic portosystemic shunt. Radiology 1995;197:805-11.

34 Schäfer C, Zundler J, Bode JC: Thrombolytic therapy in patients with portal vein thrombosis: case report and review of literature. Eur J Gastroenterol Hepatol 2000;12:1141-45.

35 Ludwig DJ, Hauptmann E, Rosoff L Jr, Neuzil D: Mesenteric and portal vein thrombosis in a young patient with protein $\mathrm{S}$ deficiency treated with urokinase via the superior mesenteric artery. J Vasc Surg 1999;30:551-4.

36 Kim DI, Lee BB, Noh SI, Baek YH, Joh JW, Lee SK, Kim YI: Conservative management of superior mesenteric and portal vein thrombosis, associated with protein $\mathrm{C}$ and $\mathrm{S}$ deficiency. Case report. Int Angiol 1997;16:235-8.

37 Ng PC, Ashari L: Portal verin thrombosis following laparoscopic surgery in a patient with sickle cell disease. Surg Endosc 2003;17:831. 